\title{
ON THE EXPONENTIAL MAPS AND THE TRIANGULAR 2-CHOMOLOGY OF GRADED LIE RINGS OF LENGTH THREE
}

\author{
HISASI MORIKAWA
}

1. Let $H$ be a group. We mean by an $N$-series in $H$ a decreasing series of subgroups $H_{1}=H, H_{2}, \ldots, H_{n+1}=\{e\}$ such that the commutator $x y x^{-1} y^{-1}$ of two elements $x$ and $y$ respectively in $H_{i}$ and $H_{j}$ belongs to $H_{i+j}$, where $H_{s}=\{e\}$ for $s \geq n+1$. We call $n$ the length of the $N$-series $\left(H_{i}\right)$. We mean by a graded Lie ring of length $n$ a Lie ring $\&$ which is a direct sum $A_{1}+\cdots$ $+A_{n}$ of additive subgroups $A_{1}, \ldots, A_{n}$ such that $\left[A_{i}, A_{j}\right] \subset A_{i+j}$, where $A_{s}=\{0\}$ for $s \geq n+1$. For each $N$-series $\left(H_{i}\right)$ of length $n$ the graded Lie ring $\mathbb{R}\left[\left(H_{i}\right)\right]$ is associated with $\left(H_{i}\right)$ as follows ${ }^{11}$ :

1) $\mathfrak{R}\left[\left(H_{i}\right)\right]$ is the direct sum of the additively written factor groups $A_{i}$ $=H_{i} / H_{i+1}(i=1,2, \ldots, n)$, and this direct sum gives the addition in $\mathfrak{Q}\left[\left(H_{i}\right)\right]$.

2) The Lie product $[a, b]$ of $a \in A_{i}$ and $b \in A_{j}$ is the group commutator $x y x^{-1} y^{-1}$ modulo $H_{i+j+1}$ of the representatives $x$ and $y$ respectively of $a$ and $b$ in $H_{1}$. We shall call $\&\left[\left(H_{i}\right)\right]$ the graded Lie ring associated with the $N$ series $\left(H_{i}\right)$.

In the present note we shall introduce triangular 2-cocycles of a graded Lie ring $\mathfrak{L}$ of length three and shall show that for each triangular 2-cocycle $\gamma$ of $\mathbb{Z}$ we can define the Exponential Map $\operatorname{Exp}_{r}$ of $\mathfrak{L}$ (onto a group) that is a bijective map of $\mathbb{Q}$ such that 1) $H_{1}=\operatorname{Exp}_{\gamma}\left(A_{1}+A_{2}+A_{3}\right), H_{2}=\operatorname{Exp}_{\gamma}\left(A_{2}+A_{3}\right)$, $H_{3}=\operatorname{Exp}_{\boldsymbol{\gamma}}\left(A_{3}\right), H_{4}=\{e\}$ form an $N$-series and 2) $\mathfrak{L}$ is regarded as the graded Lie ring $\mathfrak{R}\left[\left(H_{i}\right)\right]$ associated with $\left(H_{i}\right)$. Two triangular 2 -cocycles $\gamma$ and $\gamma^{\prime}$ are called to be equivalent if the corresponding $N$-series $\left(H_{i}\right)$ and $\left(H_{i}^{\prime}\right)$ are isomorphic. We shall call the equivalent classes of triangular 2-cocycles the triangular cohomology classes of $\mathbb{L}$. We shall also show that for any $N$-series $\left(H_{i}\right)$ of length three there exists a triangular 2 -cocycle $\beta$ of $\&\left[\left(H_{i}\right)\right]$ such that the $N$-series corresponding to the pair $\left.\left(\Omega\left[H_{i}\right)\right], \beta\right)$ is isomorphic to $\left(H_{i}\right)$. So

Received September 18, 1962.

1) See [1] 18. 4 p. 329 . 
we can conclude that the set of $N$-series of length three corresponds bijectively to the set of pairs consisting of a graded Lie ring $\mathfrak{L}$ of length three and a triangular 2-cohomology class of $\mathfrak{L}$. This is a generalization of theory of central extensions of abelian groups by abelian groups.

2. Triangular 2-cocycles. Let $\mathfrak{Q}=A_{1}+A_{2}+A_{3}$ be a graded Lie ring of length three. We regard $A_{j}$ as an $A_{i}$-module on which $A_{i}$ operates simply, and denote by $C^{2}\left(A_{i}, A_{j}\right)$ the additive group of 2 -cochains of $A_{i}$ with coefficients in $A_{j}$. We mean by a triangular 2 -cochain a triangular matrix

$$
\gamma=\left(\begin{array}{ll}
\gamma_{21} & 0 \\
\gamma_{31} & \gamma_{32}
\end{array}\right)
$$

with components $\gamma_{j i}$ in $C^{2}\left(A_{i}, A_{j}\right),(i<j)$. We denote by $C^{2}(L)$ the set of triangular 2-cochains of $L$. For $a=a_{1}+a_{2}+a_{3}, b=b_{1}+b_{2}+b_{3} \quad\left(a_{i}, b_{i} \in A_{i} ; i=\right.$ $1,2,3)$ and $\gamma \in C^{2}(\&)$ we mean by $\gamma(a, b)$ the triangular matrix

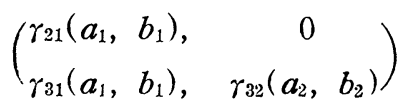

We shall now define triangular 2-cocycles of $\mathfrak{Q}$ :

Definition. A triangular 2-cocycle of $\mathfrak{Z}$ is a triangular 2 -cochain $\gamma$ of $\mathfrak{Q}$ satisfying

$$
\begin{aligned}
& \partial \gamma_{21}=0, \partial \gamma_{32}=0^{2)} \text {, } \\
& \partial \gamma_{31}\left(a_{1}, b_{1}, c_{1}\right)+\left[a_{1}, \gamma_{21}\left(b_{1}, c_{1}\right)\right] \\
& +\gamma_{32}\left(\gamma_{21}\left(b_{1}, c_{1}\right), \gamma_{21}\left(a_{1}, b_{1}+c_{1}\right)\right)-\gamma_{32}\left(\gamma_{21}\left(a_{1}, b_{1}\right), \gamma_{21}\left(a_{1}+b_{1}, c_{1}\right)\right)=0 \text {, } \\
& \left(a_{1}, b_{1}, c_{1} \in A_{1}\right) \text {, } \\
& \gamma(0, a)=\gamma(a, 0)=0,(a \in \mathbb{Q}), \\
& \gamma_{21}\left(a_{1}, b_{1}\right)-\gamma_{21}\left(b_{1}, a_{1}\right)=\left[a_{1}, b_{1}\right] \text {, } \\
& \left(a_{1}, b_{1} \in A_{1}\right) \text {, } \\
& \gamma_{32}\left(a_{2}, b_{2}\right)=\gamma_{32}\left(b_{2}, a_{2}\right),\left(a_{2}, b_{2} \in A_{2}\right) \text {. }
\end{aligned}
$$

Lemma. Let $\gamma$ be a triangular 2-cocycle of $\mathfrak{S}$. Then

$$
\begin{array}{r}
\gamma_{31}\left(a_{1},-a_{1}\right)-\gamma_{31}\left(-a_{1}, a_{1}\right)=\left[\begin{array}{rr}
a_{1}, & \gamma_{21}\left(-a_{1}, a_{1}\right)
\end{array}\right], \\
\left(a_{1} \in A_{1}\right),
\end{array}
$$

Proof. From (1), (2), (3) it follows

2) We mean by $\partial$ the ordinary coboundary operator. 


$$
\begin{aligned}
0= & \partial \gamma_{31}\left(a_{1},-a_{1}, a_{1}\right)+\left[a_{1}, \gamma_{21}\left(-a_{1}, a_{1}\right)\right] \\
& +\gamma_{32}\left(\gamma_{21}\left(-a_{1}, a_{1}\right), \gamma_{21}\left(a_{1}, 0\right)\right)-\gamma_{32}\left(\gamma_{21}\left(a_{1},-a_{1}\right), \gamma_{21}\left(0, a_{1}\right)\right) \\
= & \gamma_{31}\left(-a_{1}, a_{1}\right)-\gamma_{31}\left(a_{1},-a_{1}\right) \\
& +\left[a_{1}, \gamma_{21}\left(-a_{1}, a_{1}\right)\right],\left(a_{1} \in A_{1}\right) .
\end{aligned}
$$

This proves $(6)$.

3. The Exponential Map associated to a triangular 2-cocycle. Let $\gamma$ be a triangular 2-cocycle of \&. For $a_{i}, b_{i} \in A_{i}(i=1,2,3)$, put

$$
\begin{aligned}
& \eta_{\gamma}\left(a_{1}+a_{2}+a_{3}, b_{1}+b_{2}+b_{3}\right)=\gamma_{21}\left(a_{1}, b_{1}\right)+\gamma_{31}\left(a_{1}, b_{1}\right)+\gamma_{32}\left(a_{2}, b_{2}\right) \\
& \quad+\left[a_{1}, b_{2}\right]+\gamma_{32}\left(a_{2}+b_{2}, \gamma_{21}\left(a_{1}, b_{1}\right)\right)
\end{aligned}
$$

and

$$
\begin{aligned}
\rho_{\gamma}\left(a_{1}+a_{2}+a_{3}\right)=c_{1}+c_{2}+c_{3}, & \\
c_{1} & =-a_{1}, c_{2}=-a_{2}-\gamma_{21}\left(a_{1}, c_{1}\right), \\
c_{3} & =-a_{3}-\gamma_{31}\left(a_{1}, c_{1}\right)-\gamma_{32}\left(a_{2}, c_{2}\right)-\left[a_{1}, c_{2}\right]-\gamma_{32}\left(a_{2}+c_{2}, \gamma_{21}\left(a_{1}, c_{1}\right)\right) .
\end{aligned}
$$

We shall first show the following properties of $\eta_{\gamma}$ and $\rho_{\gamma}$.

PROPOSITION 1. Let $\gamma$ be a triangular 2-cocycle of 2 . Then

$$
\begin{aligned}
& \eta_{\gamma}(a, 0)=\eta_{\gamma}(0, a)=0, \quad(a \in \mathfrak{L}), \\
& \eta_{\gamma}(b, c)-\eta_{\gamma}\left(a+b+\eta_{\gamma}(a, b), c\right)+\eta_{\gamma}\left(a, b+c+\eta_{\gamma}(b, c)\right)-\eta_{\gamma}(a, b)=0, \\
& \quad(a, b, c \in \mathbb{L}), \\
& a+\rho_{\gamma}(a)+\eta_{\gamma}\left(\rho_{\gamma}(a), a\right)=a+\rho_{\gamma}(a)+\eta_{\gamma}\left(a, \rho_{\gamma}(a)\right)=0, \quad(a \in \mathfrak{L}) .
\end{aligned}
$$

Proof. By virtue of (3), (7) it follows $\eta_{\gamma}(0, a)=\eta_{\gamma}(a, 0)=0,(a \in \mathfrak{Q})$. By virtue of (1), (2), (3), (6), (7) for $a=a_{1}+a_{2}+a_{3}, b=b_{1}+b_{2}+b_{3}, c=c_{1}+c_{2}+c_{3}$, $\left(a_{i}, b_{i}, c_{i} \in A_{i} ; i=1,2,3\right)$, we have

$$
\begin{aligned}
& \eta_{r}(b, c)-\eta_{r}\left(a+b+\eta_{r}(a, b), c\right)+\eta_{\gamma}\left(a, b+c+\eta_{r}(b, c)\right)-\eta_{\gamma}(a, b) \\
&= \partial \gamma_{21}\left(a_{1}, b_{1}, c_{1}\right)+\partial \gamma_{31}\left(a_{1}, b_{1}, c_{1}\right)+\gamma_{32}\left(b_{2}, c_{2}\right)-\gamma_{32}\left(a_{2}+b_{2}+\gamma_{21}\left(a_{1}, b_{1}\right), c_{2}\right) \\
&+\gamma_{32}\left(a_{2}, b_{2}+c_{2}+\gamma_{21}\left(b_{1}, c_{1}\right)\right)-\gamma_{32}\left(a_{2}, b_{2}\right)+\left[b_{1}, c_{2}\right]-\left[a_{1}+b_{1}, c_{2}\right] \\
&+\left[a_{1}, b_{2}+c_{2}+\gamma_{21}\left(b_{1}, c_{1}\right)\right]-\left[a_{1}, b_{2}\right] \\
&+\gamma_{32}\left(\left(b_{2}+c_{2}, \gamma_{21}\left(b_{1}, c_{1}\right)\right)-\gamma_{32}\left(a_{2}+b_{2}+c_{2}+\gamma_{21}\left(a_{1}, b_{1}\right), \gamma_{21}\left(a_{1}+b_{1}, c_{1}\right)\right)\right. \\
&+\gamma_{32}\left(a_{2}+b_{2}+c_{2}+\gamma_{21}\left(b_{1}, c_{1}\right), \gamma_{21}\left(a_{1}, b_{1}+c_{1}\right)\right)-\gamma_{32}\left(a_{2}+b_{2}, r_{21}\left(a_{1}, b_{1}\right)\right) \\
&= \partial \gamma_{31}\left(a_{1}, b_{1}, c_{1}\right)+\left[a_{1}, \gamma_{21}\left(b_{1}, c_{1}\right)\right] \\
&+\gamma_{32}\left(a_{2}+b_{2}+c_{2}, \gamma_{21}\left(b_{1}, c_{1}\right)\right)-\gamma_{32}\left(a_{2}+b_{2}+c_{2}, \gamma_{21}\left(a_{1}, b_{1}\right)\right) \\
&+\gamma_{32}\left(a_{2}+b_{2}+c_{2}+\gamma_{21}\left(b_{1}, c_{1}\right), \gamma_{21}\left(a_{1}, b_{1}+c_{1}\right)\right)-\gamma_{32}\left(a_{2}+b_{2}+c_{2}+\gamma_{21}\left(a_{1}, b_{1}\right),\right. \\
&\left.\gamma_{21}\left(a_{1}+b_{1}, c_{1}\right)\right)
\end{aligned}
$$




$$
\begin{aligned}
= & \partial \gamma_{31}\left(a_{1}, b_{1}, c_{1}\right)+\left[a_{1}, \gamma_{21}\left(b_{1}, c_{1}\right)\right] \\
& +\gamma_{32}\left(a_{2}+b_{2}+c_{2}, \gamma_{21}\left(a_{1}, b_{1}+c_{1}\right)+\gamma_{21}\left(b_{1}+c_{1}\right)\right)+\gamma_{32}\left(\gamma_{21}\left(a_{1}, b_{1}+c_{1}\right),\right. \\
& \left.\gamma_{21}\left(b_{1}, c_{1}\right)\right) \\
& -\gamma_{32}\left(a_{2}+b_{2}+a_{2}, \gamma_{21}\left(a_{1}+b_{1}, c_{1}\right)+\gamma_{21}\left(a_{1}, b_{1}\right)\right)-\gamma_{32}\left(\gamma_{21}\left(a_{1}+b_{1}, c_{1}\right),\right. \\
\gamma_{21}\left(a_{1}, b_{1}\right) & \\
= & \partial \gamma_{31}\left(a_{1}, b_{1}, c_{1}\right)+\left[a_{1}, \gamma_{21}\left(b_{1}, c_{1}\right)\right] \\
& +\gamma_{32}\left(\gamma_{21}\left(b_{1}, c_{1}\right), \gamma_{21}\left(a_{1}, b_{1}+c_{1}\right)\right)-\gamma_{32}\left(\gamma_{21}\left(a_{1}, b_{1}\right), \gamma_{21}\left(a_{1}+b_{1}, c_{1}\right)\right)=0 .
\end{aligned}
$$

This proves (10). From the definition (8) of $\rho_{\gamma}$, putting $\rho_{\gamma}(a)=c_{1}+c_{2}+c_{3}$ ( $c_{i} \in A_{i}: i=1,2,3$ ), we have

$$
\begin{gathered}
a+\rho_{\gamma}(a)+\eta_{\gamma}\left(a, \rho_{\gamma}(a)\right)=\left(a_{1}+a_{2}+a_{3}\right)+\left(c_{1}+c_{2}+c_{3}\right)+\gamma_{21}\left(a_{1}, c_{1}\right) \\
\quad+\gamma_{31}\left(a_{1}, c_{1}\right)+\gamma_{32}\left(a_{2}, c_{2}\right)+\left[a_{1}, c_{2}\right]+\gamma_{32}\left(a_{2}+c_{2}, \gamma_{21}\left(a_{1}, c_{1}\right)\right)=0, \\
a+\rho_{\gamma}(a)+\eta_{\gamma}\left(\rho_{\gamma}(a), a\right)=a+\rho_{\gamma}(a)+\eta_{\gamma}\left(a, \rho_{\gamma}(a)\right)+\eta_{\gamma}\left(\rho_{\gamma}(a), a\right)-\eta_{\gamma}\left(a, \rho_{\gamma}(a)\right) \\
\quad=\eta_{\gamma}\left(\rho_{\gamma}(a), a\right)-\eta_{\gamma}\left(a, \rho_{\gamma}(a)\right)=\gamma_{21}\left(c_{1}, a_{1}\right)-\gamma_{21}\left(a_{1}, c_{1}\right)+\gamma_{32}\left(c_{2}, a_{2}\right)-\gamma_{32}\left(a_{2}, c_{2}\right) \\
\quad+\gamma_{31}\left(c_{1}, a_{1}\right)-\gamma_{31}\left(a_{1}, c_{1}\right)+\left[c_{1}, a_{1}\right]-\left[a_{1}, c_{2}\right]+\gamma_{32}\left(a_{2}+c_{2}, \gamma_{21}\left(c_{1}, a_{1}\right)\right) \\
-\gamma_{32}\left(a_{2}+c_{2}, \gamma_{21}\left(a_{1}, c_{1}\right)\right) .
\end{gathered}
$$

Since $c_{1}=-a_{1}$ and $c_{2}=-a_{2}-\gamma_{21}\left(a_{1},-a_{1}\right)$, by virtue of $(5),(6)$ we have

$$
\gamma_{21}\left(a_{1}, c_{1}\right)=\gamma_{21}\left(c_{1}, a_{1}\right), \gamma_{32}\left(c_{2}, a_{2}\right)=\gamma_{32}\left(a_{2}, c_{2}\right),
$$

and

$$
\begin{aligned}
& \gamma_{31}\left(c_{1}, a_{1}\right)-\gamma_{31}\left(a_{1}, c_{1}\right)+\left[c_{1}, a_{2}\right]-\left[a_{1}, c_{2}\right] \\
& \quad=\gamma_{31}\left(-a_{1}, a_{1}\right)-\gamma_{31}\left(a_{1},-a_{1}\right)+\left[-a_{1}, a_{2}\right]-\left[a_{1},-a_{2}-\gamma_{21}\left(a_{1},-a_{1}\right)\right] \\
& \quad=\gamma_{31}\left(-a_{1}, a_{1}\right)-\gamma_{31}\left(a_{1},-a_{1}\right)+\left[a_{1}, \gamma_{21}\left(a_{1},-a_{1}\right)\right]=0 .
\end{aligned}
$$

Hence $a+\rho_{\gamma}(a)+\eta_{\gamma}\left(\rho_{\gamma}(a), a\right)=0$. This completes the proof of Proposition 1 .

For each triangular 2-cocycle $\gamma$ of $\mathfrak{Q}$ we shall define the set of symbols $\left\{\operatorname{Exp}_{y}(a) \mid a \in \mathbb{R}\right\}$ with the following law of composition:

$$
\operatorname{Exp}_{\boldsymbol{r}}(a) \operatorname{Exp}_{\boldsymbol{\gamma}}(b)=\operatorname{Exp}_{\boldsymbol{\gamma}}\left(a+b+\eta_{\boldsymbol{\gamma}}(a, b)\right), \quad(a, b \in \mathfrak{Q}) .
$$

We call the map Expr the Exponential Map of $\mathfrak{L}$ with a base $\gamma$, and call $\gamma$ the basic triangular 2-cocycle of the Exponential Map Expr.

Proposition 2. $\operatorname{Exp}_{\gamma}(\mathbb{R})=\left\{\operatorname{Exp}_{\gamma}(a) \mid a \in \mathfrak{R}\right\}$ is a group and $\operatorname{Exp}_{\gamma}$ is a bijective map of $\mathfrak{Q}$ onto $\operatorname{Exp}_{r}(\mathfrak{Q})$.

Proof. By virtue of (9) we have $\operatorname{Exp}_{\gamma}(a) \operatorname{Exp}_{\gamma}(0)=\operatorname{Exp}_{\gamma}(0) \operatorname{Exp}_{\gamma}(a)=$ $\operatorname{Exp}_{r}(a)$, and thus $\operatorname{Exp}_{r}(0)$ is the unit element. From (11) it tollows $\operatorname{Exp}_{r}(a)$. 
$\operatorname{Exp}_{\gamma}\left(\rho_{\gamma}(a)\right)=\operatorname{Exp}_{\gamma}\left(\rho_{\gamma}(a)\right) \operatorname{Exp}_{\gamma}(a)=\operatorname{Exp}_{\gamma}(0)$. This shows that $\operatorname{Exp}_{\gamma}\left(\rho_{\gamma}(a)\right)$ is the inverse element of $\operatorname{Exp}_{r}(a)$. From (10) it follows

$$
\begin{aligned}
& \left(\operatorname{Exp}_{\gamma}(a) \operatorname{Exp}_{\gamma}(b)\right) \operatorname{Exp}_{\gamma}(c)=\operatorname{Exp}_{\gamma}\left(a+b+\eta_{\gamma}(a, b)\right) \operatorname{Exp}_{\gamma}(c) \\
& =\operatorname{Exp}_{\gamma}\left(a+b+c+\eta_{\gamma}(a, b)+\eta_{\gamma}\left(a+b+\eta_{\gamma}(a, b), c\right)\right. \\
& =\operatorname{Exp}_{\gamma}\left(a+b+c+\eta_{\gamma}(b, c)+\eta_{\gamma}\left(a, b+c+\eta_{\gamma}(b, c)\right)\right. \\
& =\operatorname{Exp}_{\gamma}(a)\left(\operatorname{Exp}_{\gamma}\left(b+c+\eta_{\gamma}(b, c)\right)=\operatorname{Exp}_{\gamma}(a)\left(\operatorname{Exp}_{\gamma}(b) \operatorname{Exp}_{\gamma}(c)\right) .\right.
\end{aligned}
$$

This shows that $\operatorname{Exp}_{r}(\mathfrak{L})$ satisfies the associative law. Therefore $\operatorname{Exp}_{r}(\mathfrak{L})$ is a group. Since $\operatorname{Exp}_{r}(a)$ are symbols, we have $\operatorname{Exp}_{r}(a)=\operatorname{Exp}_{r}(b)$ if and only if $a=b$. This completes the proof of Proposition 2 .

We mean by the Logarithmic Map $\log _{\gamma}$ the inverse map of the Exponential Map $\operatorname{Exp}_{\gamma}$. Namely $\log _{\gamma}$ is a bijective map of $\operatorname{Exp}_{\gamma}(\&)$ onto $\&$ such that

$$
\log _{\gamma}\left(\operatorname{Exp}_{\gamma}(a)\right)=a, \quad(a \in \mathfrak{L}) .
$$

Proposition 3. For any $a_{i}, b_{i} \in A_{i}(i=1,2,3)$ we have

$$
\text { (14) } \begin{aligned}
& \log _{\gamma}\left(\operatorname{Exp}_{\gamma}\left(a_{1}+a_{2}+a_{3}\right) \operatorname{Exp}_{\gamma}\left(b_{1}+b_{2}+b_{3}\right)\right)=\left(a_{1}+b_{1}\right)+\left(a_{2}+b_{2}+\gamma_{21}\left(a_{1}, b_{1}\right)\right) \\
&+\left(a_{3}+b_{3}+\gamma_{31}\left(a_{1}, b_{1}\right)+\gamma_{32}\left(a_{2}, b_{2}\right)+\left[a_{1}, b_{2}\right]+\gamma_{32}\left(a_{2}+b_{2}, \gamma_{21}\left(a_{1}, b_{1}\right)\right)\right), \\
&(15) \quad \log _{\gamma}\left(\operatorname{Exp}_{\gamma}\left(a_{1}+a_{2}+a_{3}\right)^{-1}\right)=\log _{\gamma}\left(\rho_{\gamma}\left(a_{1}+a_{2}+a_{3}\right)\right) \\
&=-a_{1}-\left(a_{2}+\gamma_{21}\left(a_{1},-a_{1}\right)-\left(a_{3}+\gamma_{31}\left(a_{1},-a_{1}\right)\right.\right. \\
& \quad+\gamma_{32}\left(a_{2},-a_{2}-\gamma_{21}\left(a_{1},-a_{1}\right)\right) \\
&\left.+\left[a_{1},-a_{2}-\gamma_{21}\left(a_{1}, a_{1}\right)\right]+\gamma_{32}\left(-\gamma_{21}\left(a_{1}, a_{1}\right), \gamma_{21}\left(a_{1},-a_{1}\right)\right)\right) .
\end{aligned}
$$

Proof. Since $\operatorname{Exp}_{\gamma}\left(a_{1}+a_{2}+a_{3}\right) \operatorname{Exp}_{\gamma}\left(b_{1}+b_{2}+b_{3}\right)=\operatorname{Exp}_{\gamma}\left(a_{1}+b_{1}+a_{2}+b_{2}+a_{3}\right.$ $+b_{3}+\eta_{r}\left(a_{1}+a_{2}+a_{3}, b_{1}+b_{2}+b_{3}\right)$. (14) follows from (7). (15) is an immediate consequence from (8).

For the sake of simplicity we denote by $B_{1}, B_{2}, B_{3}, B_{3}, B_{4}$ the ideals of $\mathfrak{Q}=A_{1}+A_{2}+A_{\mathrm{o}}, A_{2}+A_{3}, A_{3},\{0\}$, respectively.

Proposition 4. If $a \in B_{i}$ and $b \in B_{j}$, we have

$$
\begin{aligned}
& \log _{\gamma}\left(\operatorname{Exp}_{\gamma}(a) \operatorname{Exp}_{\gamma}(b)\right) \in B_{\min (i, j)} \\
& \log _{\gamma}\left(\operatorname{Exp}_{\gamma}(a)^{-1}\right) \in B_{i}, \\
& \log _{\gamma}\left(\operatorname{Exp}_{\gamma}(a) \operatorname{Exp}_{\gamma}(b) \operatorname{Exp}_{\gamma}(a)^{-1} \operatorname{Exp}_{\gamma}(b)^{-1}\right) \equiv[a, b] \bmod B_{i+j+1},
\end{aligned}
$$

where $B_{s}=\{0\}$ for $s \geq 4$.

Proof. (16) and (17) are immediate consequences of (14) and (15), respectively. By virtue of (14), if $a \in \mathfrak{Q}$ and $b_{3} \in A_{3}$, we have $\operatorname{Exp}_{\gamma}(a) \operatorname{Exp}_{\gamma}\left(b_{3}\right)$ 
$=\operatorname{Exp}_{r}\left(a+b_{3}\right)=\operatorname{Exp}_{r}\left(b_{3}\right) \operatorname{Exp}_{r}(a) . \quad$ This shows that $\operatorname{Exp}_{r}\left(A_{3}\right)$ is a subgroup contained in the center of $\operatorname{Exp}_{r}(\&)$. Using this fact and (1), (3), (4), (14), (15) we have for $a=a_{1}+a_{2}+a_{3}, b=b_{1}+b_{2}+b_{3}\left(a_{i}, b_{i} \in A_{i}\right)$

$$
\begin{aligned}
& \log _{r}\left(\operatorname{Exp}_{r}(a) \operatorname{Exp}_{\gamma}(b) \operatorname{Exp}_{r}(a)^{-1} \operatorname{Exp}_{r}(b)^{-1}\right) \\
& \equiv \gamma_{21}\left(a_{1}, b_{1}\right)-\gamma_{21}\left(a_{1},-a_{1}\right) \gamma_{21}\left(b_{1},-b_{1}\right)+\gamma_{21}\left(-a_{1},-b_{1}\right)+\gamma_{21}\left(a_{1}+b_{1},-a_{1}-b_{1}\right) \\
& \equiv \gamma_{21}\left(a_{1}, b_{1}\right)-\gamma_{21}\left(b_{1}, a_{1}\right) \equiv\left[a_{1}, b_{1}\right] \bmod A_{3} .
\end{aligned}
$$

This proves (16) for $i=j=1$. Since $\operatorname{Exp}_{r}\left(A_{3}\right)$ is contained in the center, (16) is also true for $i=3$ or $j=3$. So it is sufficient to prove (16) for $a=a_{2}$ and $b=b_{1}+b_{2}\left(a_{2}, b_{2} \in A_{2}, b_{1} \in A_{1}\right)$. Using (14), (15), (6) we have

$$
\begin{array}{r}
\log _{\gamma}\left(\operatorname{Exp}_{\gamma}\left(a_{2}\right) \operatorname{Exp}_{\gamma}\left(b_{1}+b_{2}\right) \operatorname{Exp}_{\gamma}\left(a_{2}\right)^{-1} \operatorname{Exp}_{\gamma}\left(b_{1}+b_{2}\right)^{-1}\right)=\log _{\gamma}\left(\operatorname{Exp}_{\gamma}\left(a_{2}\right) \operatorname{Exp}_{\gamma}\right. \\
\left.\left(b_{1}+b_{2}\right)\left(\operatorname{Exp}_{\gamma}\left(b_{1}+b_{2}\right) \operatorname{Exp}_{\gamma}\left(a_{2}\right)\right)^{-1}\right) \\
=\log _{\gamma}\left(\operatorname{Exp}_{r}\left(b_{1}+a_{2}+b_{2}+\gamma_{32}\left(a_{2}, b_{2}\right)\right) \operatorname{Exp}_{\gamma}\left(b_{1}+a_{2}+b_{2}+\gamma_{32}\left(b_{2}, a_{2}\right)+\left[b_{1}, a_{2}\right)^{-1}\right)\right. \\
=\log _{\gamma}\left(\operatorname{Exp}_{\gamma}\left(b_{1}+a_{2}+b_{2}+\gamma_{32}\left(a_{2}, b_{2}\right)\right) \operatorname{Exp}_{\gamma}\left(b_{1}+a_{2}+b_{2}+\gamma_{32}\left(a_{2}, b_{2}\right)\right)^{-1}\right. \\
\left.\operatorname{Exp}_{r}([b, a])^{-1}\right) \\
=-\left[b_{1}, a_{2}\right]=\left[a_{2}, b_{1}\right] .
\end{array}
$$

This completes the prove of (16).

We shall now sum up the results in this paragraph in the following theorem.

ThEOREM 1. Let $\gamma$ be a triangular 2-cocycle of a graded Lie ring of length three $\mathfrak{L}=A_{1}+A_{2}+A_{3}$. Then $H_{1}=\operatorname{Exp}_{y}\left(A_{1}+A_{2}+A_{3}\right), H_{2}=\operatorname{Exp}_{\gamma}\left(A_{2}+A_{3}\right), H_{3}$ $=\operatorname{Exp}_{r}\left(A_{3}\right), H_{1}=\{e\}$ form an $N$-series such that $\mathfrak{Q}$ is regarded as the graded Lie ring associated with $\left(H_{i}\right)$.

Proof. (16), (17) and (18) in Proposition 4 show that $H_{1}, H_{2}, H_{3}, H_{4}$ form an $N$-series. Identifying $\operatorname{Exp}_{\gamma}\left(a_{i}\right) \bmod H_{i+1}$ with $a_{i} \bmod B_{i+1}$, we get the identification of $\mathfrak{Q}\left[\left(H_{i}\right)\right]$ with $\mathfrak{Q}$.

4. In this paragraph we shall prove the following theorem:

Theorem 2. Let $H_{1}=H, H_{2}, H_{3}, H_{4}=\{e\}$ be an $N$-series. Then there exists a triangular 2-cocycle $\gamma$ of the graded Lie ring $\mathbb{R}\left[\left(H_{i}\right)\right]$ associated with $\left(H_{i}\right)$ such that the $N$-series associated with the pair $\left(\mathfrak{S}\left[\left(H_{i}\right)\right], \gamma\right)$ (in the sense of Theorem 1) is isomorphic to the $N$-series $\left(H_{i}\right)$.

In the proof of Theorem 2, we shall also show the structural meaning of the triangular 2 -cocycle $\gamma$. 
The proof of Theorem 2: We denote by $\mathfrak{L}\left[\left(H_{i}\right)\right]=A_{1}+A_{2}+A_{3}$ the graded Lie ring associated with $\left(H_{i}\right)$. We shall identify $H_{i} / H_{i+1}$ with $A_{i}$ and shall use the both notations (the additive one and the multiplicative one) freely for the sake of simplicity. Let us choose a family of representatives $\left\{v_{j i}(\xi) \in H_{1} / H_{j+1} \mid\right.$ $\left.\xi \in H_{1} / H_{i+1} ; 1 \leq i \leq j \leq 3\right\}$ such that

$$
\begin{aligned}
& v_{i, i}(\xi)=\xi, \\
& v_{k, j}\left(v_{j, i}(\xi)\right)=v_{k, i}(\xi), \quad\left(\xi \in H_{1} / H_{i+1} ; 1 \leq i \leq j \leq k \leq 3\right\}, \\
& v_{k, j}\left(\eta v_{j i}(\xi)\right)=v_{k, j}(\eta) v_{k, i}(\xi), \quad\left(\xi \in H_{1} / H_{i+1} ; \eta \in H_{l} / H_{i+1} ;\right.
\end{aligned}
$$

$1 \leq i<l \leq j \leq k \leq 3)$,

Put

$$
v_{j, i}(e)=\text { the unit element } e \text { of } H_{1} / H_{j+1} \text {. }
$$

and

$$
\begin{aligned}
& c_{i-1}\left(\xi, \xi^{\prime}\right)=v_{i, i-1}(\xi) v_{i, i-1}\left(\xi^{\prime}\right) v_{i, i-1}\left(\xi \xi^{\prime}\right)^{-1}, \quad\left(\xi, \xi^{\prime} \in H_{1} / H_{i} ; i=2,3\right), \\
& \gamma_{21}\left(a_{1}, b_{1}\right)=c_{1}\left(a_{1}, b_{1}\right), \gamma_{32}\left(a_{2}, b_{2}\right)=c_{2}\left(a_{2}, b_{2}\right), \quad\left(a_{i}, b_{i} \in A_{i} ; i=1,2\right)
\end{aligned}
$$

$$
\gamma_{31}\left(a_{1}, b_{1}\right)=c_{2}\left(v_{2,1}\left(a_{1}\right), v_{2,1}\left(b_{1}\right)\right), \quad\left(a_{1}, b_{1} \in A_{1}\right) .
$$

We shall prove that the triangular 2-cochain

$$
\gamma=\left(\begin{array}{cc}
\gamma_{21} & 0 \\
\gamma_{31} & \gamma_{32}
\end{array}\right)
$$

is a triangular 2-cocycle of $\mathfrak{Q}\left[\left(H_{i}\right)\right]$. Since $\gamma_{21}$ (resp. $\gamma_{32}$ ) are the 2-cocycles associated with the extension $H_{1} / H_{3}$ (resp. $H_{2} / H_{4}$ ) of $A_{2}$ (resp. $A_{3}$ ) by $A_{1}$ (resp. $A_{2}$ ), we have $\partial \gamma_{21}=0$ (resp. $\partial \gamma_{32}=0$ ). Since $v_{j, i}(e)=$ the unit element $e$ of $H_{1} / H_{j+1}$, we have $\gamma_{j i}\left(0, a_{j}\right)=\gamma_{j i}\left(a_{i}, 0\right)=0$ for $a_{i} \in A_{i}$. Namely $\gamma(0, a)=\gamma(a, 0)$ $=0$ for $a \in \mathfrak{Q}$. Since $H_{3}$ is contained in the center of $H$, from the definition of $\gamma_{31}$ we have for $a_{1}, b_{1}, c_{1} \in A_{1}$

$$
\begin{aligned}
&\left(v_{31}\left(a_{1}\right) v_{31}\left(b_{1}\right)\right) v_{31}\left(c_{1}\right)=v_{32}\left(v_{21}\left(a_{1}\right)\right) v_{32}\left(v_{21}\left(b_{1}\right)\right) v_{31}\left(c_{1}\right) \\
&= c_{3}\left(v_{21}\left(a_{1}\right), v_{21}\left(b_{1}\right)\right) v_{32}\left(v_{21}\left(a_{1}\right) v_{21}\left(b_{1}\right)\right) v_{31}\left(c_{1}\right) \\
&= \gamma_{31}\left(a_{1}, b_{1}\right) v_{52}\left(c_{21}\left(a_{1}, b_{1}\right) v_{31}(a+b) v_{31}\left(c_{1}\right)\right) \\
&= \gamma_{31}\left(a_{1}, b_{1}\right) v_{32}\left(c_{21}\left(a_{1}, b_{1}\right)\right) \gamma_{31}\left(a_{1}+b_{1}, c_{1}\right) v_{52}\left(c_{21}\left(a_{1}+b_{1}, c_{1}\right)\right) \\
& v_{31}\left(a_{1}+b_{1}+c_{1}\right) \\
&= \gamma_{31}\left(a_{1}, b_{1}\right) \gamma_{31}\left(a_{1}+b_{1}, c_{1}\right) \gamma_{32}\left(\gamma_{21}\left(a_{1}, b_{1}\right), \gamma_{21}\left(a_{1}+b_{1}, c_{1}\right)\right) \\
& v_{32}\left(\gamma_{31}\left(a_{1}, b_{1}\right)+\gamma_{21}\left(a_{1}+b_{1}, c_{1}\right)\right) v_{31}\left(a_{1}+b_{1}+c_{1}\right)
\end{aligned}
$$

and 


$$
\begin{aligned}
& v_{31}\left(a_{1}\right)\left(v_{31}\left(b_{1}\right) v_{31}\left(c_{1}\right)\right)=v_{31}\left(a_{1}\right) \gamma_{31}\left(b_{1}, c_{1}\right) v_{32}\left(c_{2}\left(b_{1}, c_{1}\right)\right) v_{31}\left(b_{1}+c_{1}\right) \\
&= \gamma_{31}\left(b_{1}, c_{1}\right) v_{31}\left(a_{1}\right) v_{32}\left(c_{2}\left(b_{1}, c_{1}\right)\right) v_{31}\left(a_{1}\right)^{-1} v_{31}\left(c_{2}\left(b_{1}, c_{1}\right)\right)^{-1} \\
& v_{32}\left(c_{2}\left(b_{1}, c_{1}\right)\right) v_{31}\left(a_{1}\right) v_{31}\left(b_{1}+c_{1}\right) \\
&= \gamma_{31}\left(b_{1}, c_{1}\right)\left[a_{1}, \gamma_{21}\left(b_{1}, c_{1}\right)\right] v_{32}\left(c_{2}\left(b_{1}, c_{1}\right)\right) \gamma_{31}\left(a_{1}, b_{1}+c_{1}\right) \\
& v_{32}\left(c_{2}\left(a_{1}, b_{1}+c_{1}\right) v_{31}\left(a_{1}+b_{1}+c_{1}\right)\right. \\
&= \gamma_{31}\left(b_{1}, c_{1}\right) \gamma_{31}\left(a_{1}, b_{1}+c_{1}\right)\left[a_{1}, \gamma_{21}\left(b_{1}, c_{1}\right)\right] \gamma_{32}\left(\gamma_{21}\left(b_{1}, c_{1}\right), \gamma_{21}\left(a_{1}, b_{1}+c_{1}\right)\right) \\
& v_{32}\left(\gamma_{21}\left(b_{1}, c_{1}\right)+\gamma_{21}\left(a_{1}, b_{1}+c_{1}\right)\right) v_{31}\left(a_{1}+b_{1}+c_{1}\right) .
\end{aligned}
$$

Hence by the associative law and the equality $\partial r_{21}=0$ we have

$$
\begin{aligned}
\partial \gamma_{31}\left(a_{1}, b_{1}, c_{1}\right)+\left[a_{1}, \gamma_{21}\left(b_{1}, c_{1}\right)\right]+\gamma_{32}\left(\gamma_{21}\left(b_{1}, c_{1}\right), \gamma_{21}\left(a_{1}, b_{1}+c_{1}\right)\right) \\
-\gamma_{32}\left(\gamma_{21}\left(a_{1}, b_{1}\right), \gamma_{21}\left(a_{1}+b_{1}, c_{1}\right)\right)=0
\end{aligned}
$$

in the additive notation. This shows that $\gamma$ is a triangular 2 -cocycle of $\mathbb{2}\left[\left(H_{i}\right)\right]$. We denote by $\sigma$ the map of $H_{i}$ onto $\operatorname{Exp}_{\gamma}\left(\left\{\left[\left(H_{i}\right)\right]\right.\right.$ defined by

$$
\sigma\left(v_{33}\left(a_{3}\right) v_{32}\left(a_{2}\right) v_{31}\left(a_{1}\right)\right)=\operatorname{Exp}_{\gamma}\left(a_{1}+a_{2}+a_{3}\right), \quad\left(a_{i} \in A_{i}\right) .
$$

We shall shows that $\sigma$ is an isomorphism. From the definition of $\gamma$ and $\left\{v_{j i}(\xi)\right\}$ it follows

$$
\begin{aligned}
& \left(v_{33}\left(a_{3}\right) v_{32}\left(a_{2}\right) v_{31}\left(a_{1}\right)\right)\left(v_{33}\left(b_{3}\right) v_{32}\left(b_{2}\right) v_{31}\left(b_{1}\right)\right) \\
& =v_{33}\left(a_{3}\right) v_{33}\left(b_{3}\right) v_{32}\left(a_{2}\right) v_{32}\left(b_{2}\right) v_{32}\left(b_{2}\right)^{-1} v_{31}\left(a_{1}\right) v_{32}\left(b_{2}\right) v_{31}\left(a_{1}\right)^{-1} v_{31}\left(a_{1}\right) v_{31}\left(b_{1}\right) \\
& =v_{33}\left(a_{3}+b_{3}+\left[-b_{2}, a_{1}\right]\right) c_{2}\left(a_{2}, b_{2}\right) v_{32}\left(a_{2}+b_{2}\right) v_{32}\left(v_{21}\left(a_{1}\right)\right) v_{32}\left(v_{21}\left(b_{1}\right)\right) \\
& =v_{33}\left(a_{3}+b_{3}+\left[a_{1}, b_{2}\right]+\gamma_{32}\left(a_{2}, b_{2}\right)\right) v_{32}\left(a_{2}+b_{2}\right) c_{2}\left(v_{21}\left(a_{1}\right), v_{21}\left(b_{1}\right)\right) \\
& \quad v_{32}\left(v_{21}\left(a_{1}\right) v_{21}\left(b_{1}\right)\right) \\
& =v_{33}\left(a_{3}+b_{3}+\left[a_{1}, b_{2}\right]+\gamma_{32}\left(a_{2}, b_{2}\right) \gamma_{31}\left(a_{1}, b_{1}\right)\right) v_{32}\left(a_{2}+b_{2}\right) v_{32}\left(a_{1}\left(a_{1}, b_{1}\right)\right) \\
& \left.\quad v_{21}\left(a_{1}+b_{1}\right)\right) \\
& =v_{33}\left(a_{3}+b_{3}+\gamma_{31}\left(a_{1}, b_{1}\right)+\gamma_{32}\left(a_{2}, b_{2}\right)+\left[a_{1}, b_{2}\right]+\gamma_{32}\left(a_{2}+b_{2}, \gamma_{21}\left(a_{1}, b_{1}\right)\right)\right) \\
& v_{32}\left(a_{2}+b_{2}+\gamma_{21}\left(a_{1}, b_{1}\right)\right) v_{31}\left(a_{1}+b_{1}\right) .
\end{aligned}
$$

Hence by virtue of (14) in Proposition 3 we have

$$
\begin{aligned}
& \begin{array}{r}
\left(v_{33}\left(a_{3}\right) v_{32}\left(a_{2}\right) v_{31}\left(a_{1}\right) v_{33}\left(b_{3}\right) v_{32}\left(b_{2}\right) v_{31}\left(b_{1}\right)\right) \\
=\operatorname{Exp}_{\gamma}\left(a_{1}+b_{1}+a_{2}+b_{2}+\gamma_{21}\left(a_{1}, b_{1}\right)+a_{3}+b_{3}+\gamma_{31}\left(a_{1}, b_{1}\right)+\gamma_{32}\left(a_{3}, b_{2}\right)\right. \\
\left.+\gamma_{32}\left(a_{2}+b_{2}, \gamma_{21}\left(a_{1}, b_{1}\right)\right)\right)
\end{array} \\
=\operatorname{Exp}_{\gamma}\left(a_{1}+a_{2}+a_{3}\right) \operatorname{Exp}_{\gamma}\left(b_{1}+b_{2}+b_{3}\right)=\sigma\left(v_{33}\left(a_{3}\right)\right. & \left.v_{32}\left(a_{2}\right) v_{31}\left(a_{1}\right)\right) \\
& \sigma\left(v_{33}\left(b_{3}\right) v_{32}\left(b_{2}\right) v_{31}\left(b_{1}\right)\right) .
\end{aligned}
$$

This proves that $\sigma$ is a homomorphism. Since obviousely $\sigma$ is bijective, $\sigma$ is an 
isomorphism. This completes the proof of Theorem 2 .

Two triangular 2-cocycles $\gamma$ and $\gamma^{\prime}$ of $\mathscr{Z}=A_{1}+A_{2}+A_{3}$ are called to be equivalent if the $N$-series associated with the pairs $(\mathscr{R}, \gamma)$ and $\left(\mathfrak{R}, \gamma^{\prime}\right)$ (in the means of Theorem 1) are isomorphic. We call the equivalent classes of triangular 2-cocycles the triangular 2-cohomology classes of $\mathbb{2}$. Then by virtue of Theorems 1 and 2 we can conclude that the set of pairs consisting of a graded Lie ring $\mathfrak{Z}$ of length three and a triangular 2 -cohomology class of $\mathfrak{L}$ corresponds bijiectively to the set of $N$-series of length three by means of the Exponential Maps.

\section{REFERENCE}

[1] M. Hall, The Theory of Groups (1959), New York.

Supplement: Let $\mathbb{Q}=A_{1}+A_{2}+A_{3}$ be a graded Lie ring of length three. Let $\tau_{21}$ be a 2-cocycle of $A_{1}$ with coefficients in $\mathrm{A}_{2}$ and $\gamma_{32}$ be a 2-cocycle of $A_{2}$ with coefficients in $A_{3}$ such that the extended group $N_{3}$ of by $N_{2}$ with respect to $\gamma_{32}$ is abelian, i.e.

$$
0 \rightarrow A_{3} \rightarrow N_{2} \rightarrow A_{2} \rightarrow 0
$$

is an exact sequence of abelian groups. We denote by $H_{a}^{2}\left(A_{2}, A_{3}\right)$ the group of all the abelian extensions of $A_{3}$ by $A_{2}$. We denote by $\delta^{r_{32}}$ the coboundary operation of $H^{2}\left(A_{1}, A_{2}\right)$ into $H^{3}\left(A_{1}, A_{3}\right)$ with respect to the exact sequence $(*)$, then we have the following identity

$$
\begin{aligned}
\delta^{\gamma_{32}}\left(\gamma_{21}\right)\left(a_{1}, b_{1}, c_{1}\right)= & \gamma_{32}\left(\gamma_{21}\left(b_{1}, c_{1}\right), \gamma_{21}\left(a_{1}, b_{1}+c_{1}\right)\right) \\
& -\gamma_{32}\left(\gamma_{21}\left(a_{1}, b_{1}\right), \gamma_{21}\left(a_{1}+b_{1}, c_{1}\right)\right), \quad\left(a_{1}, b_{1}, c_{1} \in A_{1}\right) .
\end{aligned}
$$

On the other hand if we put $\gamma_{32}^{\prime}=\gamma_{32}+\partial \beta$ with a 1 -cochain $\beta$ of $A_{2}$, we have

$$
\delta^{\gamma_{32^{\prime}}}\left(\gamma_{21}\right)=\delta^{\gamma_{32}}\left(\gamma_{21}\right)+\partial g, g\left(a_{1}, b_{1}\right)=\beta\left(\gamma_{21}\left(a_{1}, b_{1}\right)\right), \quad\left(a_{1}, b_{1} \in A_{1}\right) .
$$

These identities show that the mapping $\left(\gamma_{32}, \gamma_{21}\right) \rightarrow \delta^{\gamma_{32}}\left(\gamma_{21}\right)$ induces the zero-map of $H_{a}^{2}\left(A_{2}, A_{3}\right) \times H^{2}\left(A_{1}, A_{2}\right)$ into $H^{3}\left(A_{1}, A_{3}\right)$. The map: $\gamma_{21} \rightarrow\left[a_{1}, \gamma_{21}\left(b_{1}, c_{1}\right)\right]$ induces a homomorphism $\chi$ of $H^{2}\left(A_{1}, A_{2}\right)$ into $H^{3}\left(A_{1}, A_{3}\right)$. We denote by $K$ the kernel of $\chi$, then we can parametrize by $K \times H_{a}^{2}\left(A_{2}, A_{3}\right) \times H^{2}\left(A_{1}, A_{3}\right)$ all $N$-series $\left\{N_{1}, N_{2}, N_{3}\right\}$ such that associated Lie ring of $\left\{N_{i}\right\}$ is canonically isomorphic to $\mathfrak{L}=A_{1}+A_{2}+A_{3}$.

Mathematical Institute, Nagoya University 